\title{
A DATA-DRIVEN DESIGN FOR DERIVING USABILITY METRICS
}

\author{
Tamara Babaian, Wendy Lucas and Heikki Topi \\ CIS Department, Bentley College, Waltham, MA 02452, USA \\ tbabaian@bentley.edu,wlucas@bentley.edu,htopi@bentley.edu
}

\begin{abstract}
Keywords: Enterprise information systems, data modeling, user input logs, usability.
Abstract: The complexity of Enterprise Information Systems can be overwhelming to users, yet they are an often overlooked domain for usability research. To better understand the ways in which users interact with these systems, we have designed an infrastructure for input logging that is built upon a data model relating system components, user inputs, and tasks. This infrastructure is aware of user representations, task representations, and the history of user interactions. The interface components themselves log user inputs, so that timing data and action events are automatically aligned and are linked to specific tasks. The knowledge gained about user interactions at varying levels of granularity, ranging from keystroke analysis to higher-level task performance, is a valuable resource for both assessing and enhancing system usability.
\end{abstract}

\section{INTRODUCTION}

Enterprise systems link processes from across the organization under one umbrella. While one of the defining characteristics of these systems is the large and well-documented set of structured tasks that they support, users can be overwhelmed by the variety of available options and the multiple paths through the system for completing processes composed of several subtasks (Topi et al., 2005). Despite being employed extensively for critical business functions, these systems often suffer from poor usability and can be very difficult to learn. In this paper, we describe our approach to leveraging information that can be derived from user input logs generated from interactions with such a system. This research is motivated by the goal of improving the design of user-system interactions and, consequently, the usability of enterprise-wide information systems.

Most of the tasks in an enterprise system are implemented as sequences of input forms. In the experimental system we have developed for studying users' interactions with enterprise systems, we explicitly represent the relationships between system tasks and the interface pages that implement them. This experimental system and the data collected from its use is exploited for high-level reasoning about sys- tem usage and usability. We show how this improved understanding of system use can also lead to various interface enhancements.

Our infrastructure enables automatic logging of user interactions with the interface and makes the log data available to the system. Input-aware components are used to track input data and associate it with the user who entered it. The model underlying this infrastructure represents and links users, tasks, interface components, application domain data, and user input logs. The input log data can be used for a variety of purposes, including deriving user profiles, modeling tasks, assessing the usability of the system, and supporting interface adaptation. Contributions of the research presented here include:

- a model that relates user interface components, user input, task structure and domain data, and

- an approach to automated analysis of system use that can be applied to systems ranging from single-user to enterprise-wide applications.

In the following sections, we describe how users and tasks performed with a system are modeled in our infrastructure and detail our component-enabled approach to user input logging. We then discuss how user profiles and models of the relationships between users and tasks can be formed from the input logs. We 
also show how the logged data can be used for deriving usability assessments and personalizing the user experience.

\section{RELATED WORK}

User input logging for subsequent log analysis was introduced a long time ago and has been used in multiple contexts. A known but infrequently used benefit from the collected data is the usability measures that can be derived from the logs without the need for any special equipment or experiments. Ivory and Hearst (Ivory and Hearst, 2001) present a review of a wide variety of usability evaluation automation techniques and discuss the challenges of these types of evaluation as well as the characteristics of the surveyed approaches. As a step towards enabling applications to automatically collect usage data, Olsen and Harversen (Olsen and Halversen, 1988) present a User Interface Management System that augments each user interface component with the capability to log user input. KALDI (Al-Qaimari and McRostie, 2006) is a graphical toolkit for automatic recording and visualization of the usage data. An implentation of the detailed tracking of users' interactions with a website via an HTTP proxy that intercepts client-server communications and extends the client application with usage logging capabilities is presented in (Atterer et al., 2006).

Usage data has been used almost exclusively for extending the functionality of automated tutoring, recommender, and adaptive hypermedia applications (e.g. (Linton et al., 2000; Brusilovsky and Cooper, 2002)). Within these types of systems, the usersystem interaction data is used for tracking and assessing the competency of the user with a topic or task and adjusting the scope, content or presentation of the material based on this assessment. Some of these systems employ a direct representation of the tasks and their hierarchical decomposition (e.g. (Brusilovsky and Cooper, 2002)) in order to relate the user model to particular tasks and their components.

The approach presented in this paper takes advantage of user logging for usability assessment and for application enhancements in the domain of enterprise systems. What distinguishes our research is the explicit representation of the task model as a composition of interface components within the system's data model. The presented model links users, tasks, interface components and the application's domain data, enabling sophisticated reasoning about the history of system usage on different levels: from focusing on a single input mechanism, to discovering user roles as a combination of frequently co-occurring tasks. An important aspect for the domain of enterprise systems is the ability afforded by this infrastructure to trace back the content of the application's domain data to the particular set of users who entered it.

\section{THE MODEL}

Users of Enterprise Resource Planning (ERP) systems and other enterprise systems enter text into text fields, select items from menus and list structures, mark check boxes, and click on links. In order to capture such inputs to a system, we have defined an experimental infrastructure in which all components that accept user input are based on a common architecture that implements logging of that input. The logged data is accessible to the system and provides a full history of the users' interactions.

Figure 1 illustrates the relationships between the users, the data they enter, the input controls being used and their groupings into task-related pages, the tasks being performed with those pages, and the domain-specific process related to those tasks. We have developed a prototype that uses this model in the implementation of a Purchase Requisition Process from an ERP system. The major components in the model are defined as follows. Domain data is the part of the model that stores the business data. User represents the user information. UI Concept includes entities representing the user interface components, their grouping on interface pages, and their relationship to the domain data. The Task Concept component represents tasks as named collections of interface pages. The Tracking component contains the entities that record the interaction history: which tasks and input controls were enacted, when, and what data was entered.

User, Task and UI Concepts form the static components of the model, i.e., they represent the data that does not change during system use. The Domain and Tracking modules are dynamic.

\subsection{Domain, User, Task and Ui Concept}

The Domain data portion of the model represents the business data, such as the description of materials, vendors, purchase requisitions and other relevant entities. This is the standard part of any information system that stores and manipulates data in support of business operations.

Multiple users are represented in the User entity.

The UI Concept module defines the composition of the interface and its connection to the domain data. The most basic entity of this module is the InputControl, which provides a specification for a user-input 


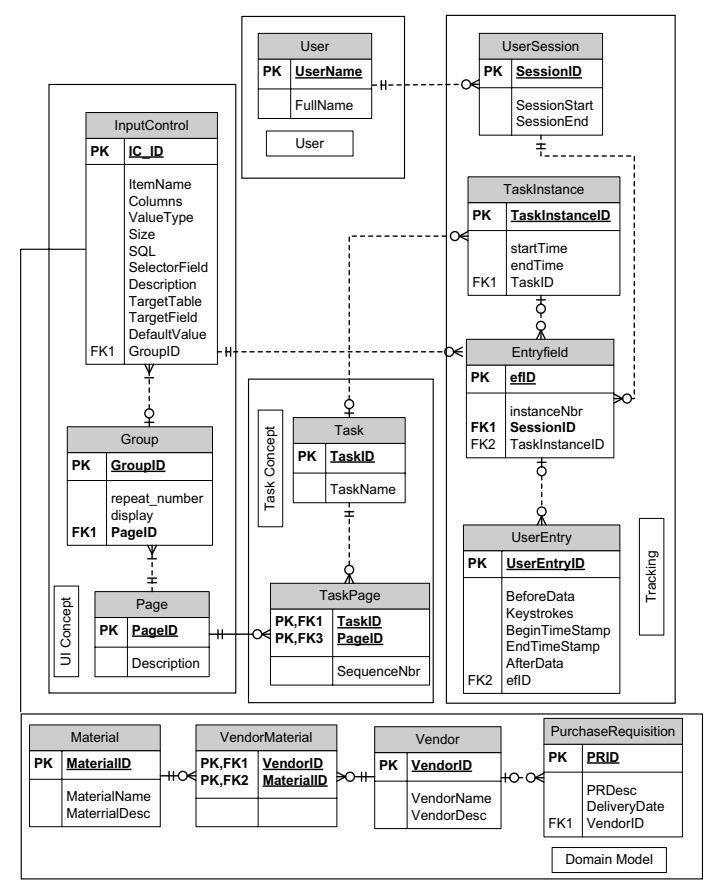

Figure 1: Components of the data model.

component. It contains attributes that define the format of the input control (ItemName, Columns, ValueType, Size, e.g.) and the way by which it is connected to (a) the domain data and (b) the task in which it is used.

To establish a relationship with the domain data, InputControl table entries specify where the data that is displayed by or entered into the component is stored in the Domain model. For example, the $S Q L$ attribute specifies the SQL code for retrieving the set of possible values for this input control, while the TargetTable and TargetField attributes specify where the values from the instances of this InputControl will be saved. Data values that are not retrieved from or stored in the Domain data, such as calendar dates, are handled as special cases.

To describe the higher level composition of the interface, InputControls form collections at two levels: Groups and Pages (i.e., distinct interface screens). InputControls are linked to Tasks through the Groups and Pages collections.

A Task Concept lists the tasks implemented by the system and describes their composition in the form of a numbered sequence of interface pages. Each page can potentially be associated with multiple tasks; therefore, the entity TaskPage is required to identify the specific tasks associated with a page.

\subsection{Tracking}

The Tracking module contains the data that is dynamically collected each time the system is invoked. It represents the history of the users' interactions with the system. Below we describe the entities of the Tracking module and the way they are populated with data when the system is being used.

When a user logs into the system, a new UserSession record is created. It records the identity of the user as well as the start time of the session in the SessionStart attribute. The end time of the session will be captured upon the log off in the SessionEnd attribute.

After the user declares his intention to work on a particular task by selecting it from a menu, the system creates a new record in the TaskInstance table that references the selected task's record in the Task table. Each TaskInstance is associated with the corresponding UserSession that links it to the user.

The system simultaneously determines the first TaskPage of the task's interface and renders the content of the page based on the specification of groups of input controls, which it obtains from the associated records in the UI component. When a widget described in the InputControl associated with the current page is created and added to the displayed interface, the system creates a record of the new instance of that InputControl in the EntryField table. This distinguishes the multiple instances of the same InputControl that can be created in the course of a single session.

Each input component created from an InputControl specification has the capability of logging user keystroke and mouse button press data directed to it. This data is periodically saved in the UserEntry table. A record in the UserEntry table specifies the time when the input component gained the focus of user input in the BeginTimeStamp attribute, the keystrokes (or mouse events) that were made (Keystrokes attribute) until the time when the widget lost focus (EndTimeStamp), and the content of the widget upon gaining and losing focus (BeforeData, AfterData). The redundancy in replicating the content of the field is deliberate and supports more efficient reasoning: instead of trying to deduce the content upon the end of the recorded interaction, we save it explicitly in the AfterData field.

To summarize, the Tracking module records user input in the form of key strokes and mouse presses in the UserEntry table, which is connected through the EntryField to specific TaskInstances and InputControls. UserEntry is also related to a particular User through EntryField and UserSession. 


\section{USING LOG DATA}

A well-known challenge in utilizing the data collected via logging information at the keystroke level is the problem of aggregating it in order to relate that data to higher level tasks (Ivory and Hearst, 2001). The meta-model presented above allows the grouping of specific data entry events along a variety of dimensions, including users, tasks, and various interface components. These groupings are possible because of the richness of the model, which links individual data entry records to interface pages, thereby providing a description of the context of each record. Furthermore, the provided description of the composition of individual tasks from user interface components allows one to derive and analyze usage patterns related to tasks. Such analysis can be extended to logged data from a group of users, providing even more information about the way the system is utilized. The results from this analysis can be applied to assessing the usability of the system and improving its design.

\subsection{Usability Metrics}

The ISO (ISO, 1998) defines usability as the effectiveness, efficiency, and satisfaction with which specified users achieve specified goals using a particular interface. Effectiveness refers to the accuracy and completeness with which the users achieve their goals. Efficiency refers to resources expended in relation to the accuracy and completeness of goals achieved, and satisfaction denotes the comfort and acceptability of the system to its users and other people affected by its use.

In the domain of enterprise systems, individual tasks represent a part of a larger business process. For example, a purchase requisition is part of the larger ordering process. A business process may span across multiple human participants and extend over multiple days. The individual tasks performed as part of a process are connected, in that the completion of one may be a prerequisite for the completion of another. Therefore, it is often useful to analyze usage data over not just one task, but over a sequence of tasks.

Once a sequence of tasks has been identified, the database structure allows us to retrieve information regarding the entire sequence of interest (based on StartTime and EndTime attributes from TaskInstance) and its individual components. The time between the component tasks can also be estimated. This allows for the detection of users suffering from navigation problems, as indicated by a large amount of time spent moving from one user interface component to another.

A detailed analysis of the rows in the UserEntry table can help identify which input components are particularly prone to erroneous data entries, corrections, extra keystrokes, and other similar user actions that indicate confusion or difficulty in understanding the requirements of a particular element of a task. An analysis at the keystroke level can also reveal inefficient methods for entering data.

\subsection{Enhancements for Imporoving Usability}

The log data provides information related to the user's familiarity and competency with the system at several levels of detail. At the lowest level, it is possible to observe how many times a person has interacted with a particular input field. At a higher level, the log data can reveal how many times a user has worked on a particular task or on a sequence of tasks. The number of times measure is an approximation for familiarity. Furthermore, a more competent user is expected to make fewer changes to an entry field and to complete a task in a shorter period of time. This information can be derived by relating data regarding user entries to particular entry fields as well as to task instances and task sequences.

Such information can then be used to dynamically personalize the system to more effectively meet the needs of the user. For example, users who typically have difficulties with particular tasks could be directed to more detailed help instructions, while experienced users could be provided with shortcuts and tips commensurate with their abilities.

The knowledge gained by analyzing an individual user's behavior can be even more valuable if augmented with knowledge of all users of the system. For example, by considering the sequences of tasks performed by a group of users, we can identify the roles of those users and determine the types of difficulties they are encountering. This will provide additional information on those parts of the interface that require attention and how the system can be enhanced to benefit users in particular roles.

As with the information on individual users, data from groups of users can be dynamically derived from the user logs. Components can then be designed to provide more (or less) assistance based on the amount of time users typically spend interacting with them. Sequences of tasks can be grouped in the interface based on paths typically followed by one user or by groups of users. Tasks that are found to be difficult for many users can have more assistance options offered with them in the short term and be redesigned in the longer term. 


\subsection{Illustrative Examples}

To validate our approach to automated input logging and analysis of log data, we have developed a prototype system that uses the model from Figure 1 to store domain, task, interface and user input tracking data. Four people who had never used this prototype were asked to work on a typical ERP task of creating a purchase requisition. The users were given a manual describing the functions of the system. The data for four purchase requisitions of relatively similar size was also provided in textual form.

Based on the log data obtained from this experiment, we can automatically derive a variety of useful views and usage measurements. For example, Figure 2 depicts the timeline that shows the sequence of subtask instances as performed by one of the users. For demonstration purposes, the figure is limited to about $70 \%$ of the actual subtask sequence. The subtasks are listed on the left-hand side in chronological order. Horizontal bars depict the timing and duration of user interactions with a particular task interface via mouse clicks and keystrokes. Horizontal gaps between the bars represent the transition time between consecutive subtasks. This visualization was created with MS Excel charting tools using data derived by aggregating and querying the tables in the UI, Task, and Tracking modules of the data model (Fig. 1).

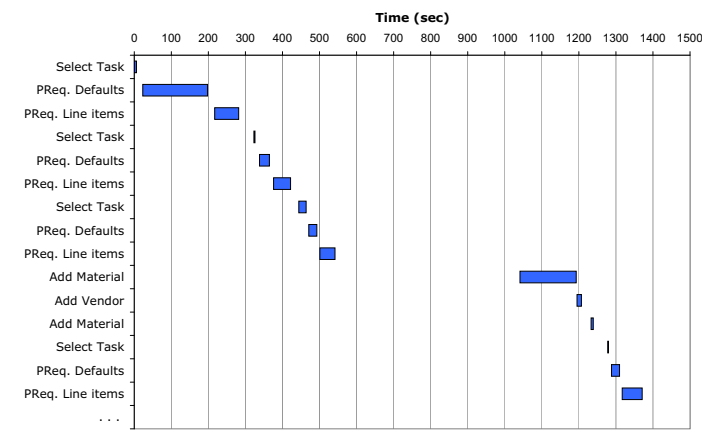

Figure 2: Chronological history of tasks performed by one of the users.

The data in the graph shows the user's interactions in creating four purchase requisitions, each beginning with a PReq. Defaults subtask. The user's first interaction with each subtask involved in the purchase requisition task tends to take longer than subsequent interactions with the same subtasks. The transition time between subtasks also follows this pattern, with that time gradually decreasing as the user gains more experience with the interface. The transition to a newly encountered requirement involving a new set of subtasks takes a significant amount of time, as evidenced by the large gap in the middle of the chart where the user moves from PReq. Line Items to Add Material.

The availability of more detailed usage data allows us to zoom in on this transition episode to further investigate what occurred during that time period. Figure 3 presents the details of the interaction around the time of this transition. The table shows the name of the subtask (TaskName column), the name of the input field to which user input was directed (ItemName column), and the field's id (efID column). The entries in the Keystrokes column designate the sequence of keystrokes or mouse press and release events. Each 〈press event for an input field triggers the display of a list of possible values from which the user can select an entry for the field. A 〈release $\rangle$ item corresponds to the closing of this list of values. For each row in the table in Figure 3, BeginTime and EndTime designate the beginning and ending timestamps for keyboard or mouse invocations. The content of the field upon the end of an interaction is shown in the AfterData column.

The data in this table allows us to reconstruct the likely sequence of events: As the user was specifying a value for the Vendor field of PReq. Line Items task interface, she invoked the display of possible values and selected a value of 3 , as evidenced by entries in the second and third row containing 〈press〉 and $\langle$ release $\rangle$ events, respectively. The user subsequently tried to add a new vendor (Office Max) by accessing the Add Material and then the Add Vendor task interfaces. The data indicates some confusion on the part of the user: before proceeding to the specification of the New Vendor Name in the Add Vendor task interface as prescribed by the user manual, she tried to enter the information regarding the new vendor through the Vendors field of the Add Material interface, as captured by row number 4 of the table. Since the Vendors input field is not designed for entering new values, the data was invalidated by the system and erased from the content of the Vendors field with efID 116. That is why the next row in table in Figure 3 (row number 5) shows an empty string in the AfterData column following a press of the mouse button.

Figure 4 is a simple illustration of the ability to aggregate data from multiple users, as afforded by our approach. It presents the breakdown of the total time spent by each of the four users on keyboard entry within the interface pages implementing the purchase requisition task versus the time spent in selection of possible values and transitions from one interface component to another. It is interesting to note that usr3, who spent the least amount of time on keyboard input, was the only one to not complete the task successfully. Even from this small set of values, one 


\begin{tabular}{|c|c|c|c|c|c|c|c|c|}
\hline \# & TaskName & ItemName & efID & Keystrokes & ElapsedTime & BeginTime & EndTime & Afterdata \\
\hline 1 & PReq. Line items & Delivery Date & 86 & $\langle$ release $\rangle$ & 0 & 527 & 527 & \\
\hline 2 & PReq. Line items & Vendor & 88 & $\langle$ press $\rangle$ & 0 & 540 & 540 & 3 \\
\hline 3 & PReq. Line items & Vendor & 88 & $\langle$ release $\rangle$ & 0 & 542 & 542 & 3 \\
\hline 4 & Add Material & Vendors & 116 & Office Max & 21 & 1041 & 1062 & Office Max \\
\hline 5 & Add Material & Vendors & 116 & 〈press $\rangle$ & 0 & 1120 & 1120 & \\
\hline 6 & Add Material & Vendors & 116 & $\langle$ release $\rangle$ & 0 & 1124 & 1124 & \\
\hline 7 & Add Material & Vendors & 116 & $\langle$ press $\rangle$ & 0 & 1131 & 1131 & \\
\hline 8 & Add Material & Vendors & 116 & $\langle$ release $\rangle$ & 0 & 1137 & 1137 & 9 \\
\hline 9 & Add Material & Vendors & 116 & $\langle\mathrm{BK}\rangle\langle\mathrm{BK}\rangle 10$ & 6 & 1137 & 1143 & 10 \\
\hline 10 & Add Material & Vendors & 116 & office max & 25 & 1173 & 1157 & office max \\
\hline 11 & Add Vendor & New Vendor Name & 121 & office max & 12 & 1195 & 1207 & office max \\
\hline 12 & Add Material & Vendors & 116 & $\langle$ press $\rangle$ & 0 & 1233 & 1233 & \\
\hline 13 & Add Material & Vendors & 116 & $\langle$ release $\rangle$ & 0 & 1239 & 1239 & \\
\hline
\end{tabular}

Figure 3: Details of chronological history. All time values are specified in seconds.

\begin{tabular}{|c|c|c|c|}
\hline User & Total Time & DataEntry Time & Difference \\
\hline usr1 & 2216 & $221(10 \%)$ & $1995(90 \%)$ \\
usr2 & 1561 & $45(3 \%)$ & $1516(97 \%)$ \\
usr3 & 1415 & $101(7 \%)$ & $1314(93 \%)$ \\
usr4 & 1815 & $136(7 \%)$ & $1679(93 \%)$ \\
\hline
\end{tabular}

Figure 4: Statistics of time spent on keyboard data entry compared to the rest of the activities involved in completing the entire task by each of the four users.

can see how useful estimates of the percentage of time spent on keyboard input can be derived. In a similar fashion, we can obtain measures estimating the transition times between tasks or between individual input fields within a given task interface.

While the examples presented here are based on a very small dataset, they illustrate the types of information that can be gleaned from user input logs that are linked via components to tasks.

\section{CONCLUSIONS}

We have described an infrastructure that supports the tracking of user input data via input-aware components and the automatic association of that data with the user who entered it, the task that required it, and the changes to the domain data resulting from it. The declarative manner in which we define the interface supports logging of user input and the subsequent attribution of that data to specific interface pages. This results in the automatic collection of usability-related data that highlights the difficulties users face in working with this type of system, the types of problems most commonly encountered, how users address their own user entry errors, and the most commonly followed navigational paths.

The next stage in this research is to conduct experimental studies using our prototype. The resulting user logs will be evaluated using the metrics described in this paper. Such assessments can then be applied to addressing shortcomings in the design, personalizing the interface, and providing better support to the user.
For enterprise systems in particular, which require substantial investments in user training, an additional benefit is the ability to focus training on those areas identified by the automated usability assessments as being most problematic for users.

\section{REFERENCES}

ISO 9241-11 (1998). Ergonomic requirements for office work with visual display terminals (VDTs) - part 11: Guidance on usability.

Al-Qaimari, G. and McRostie, D. (2006). Evaluating user performance using KALDI: A computer-aided usability engineering tool. In Proceedings of the IASTED Conf. on Software Engineering, pages 242-251.

Atterer, R., Wnuk, M., and Schmidt, A. (2006). Knowing the user's every move: user activity tracking for website usability evaluation and implicit interaction. In $W W W$, pages 203-212.

Brusilovsky, P. and Cooper, D. W. (2002). Domain, task, and user models for an adaptive hypermedia performance support system. In IUI '02: Proceedings of the 7 th international conference on Intelligent user interfaces, pages 23-30, New York, NY, USA. ACM Press.

Ivory, M. Y. and Hearst, M. A. (2001). The state of the art in automating usability evaluation of user interfaces. ACM Comput. Surv., 33(4):470-516.

Linton, F., Joy, D., Schaefer, H.-P., and Charron, A. (2000). Owl: A recommender system for organization-wide learning. Educational Technology \& Society, 3(1).

Olsen, D. R. and Halversen, B. W. (1988). Interface usage measurements in a user interface management system. In UIST '88: Proceedings of the 1st annual ACM SIGGRAPH symposium on User Interface Software, pages 102-108, New York, NY, USA. ACM Press.

Topi, H., Lucas, W., and Babaian, T. (2005). Identifying usability issues with an ERP implementation. In Proceedings of the International Conference on Enterprise Information Systems (ICEIS-2005), pages 128133. 